Homology, Homotopy and Applications, vol.5(1), 2003, pp.53-70

\title{
GROUP EXTENSIONS AND AUTOMORPHISM GROUP RINGS
}

\author{
JOHN MARTINO AND STEWART PRIDDY
}

(communicated by Lionel Schwartz)

\begin{abstract}
We use extensions to study the semi-simple quotient of the group ring $\mathbf{F}_{p} A u t(P)$ of a finite $p$-group $P$. For an extension $E: N \rightarrow P \rightarrow Q$, our results involve relations between $A u t(N)$, $\operatorname{Aut}(P), \operatorname{Aut}(Q)$ and the extension class $[E] \in H^{2}(Q, Z N)$. One novel feature is the use of the intersection orbit group $\Omega([E])$, defined as the intersection of the orbits $\operatorname{Aut}(N) \cdot[E]$ and $A u t(Q) \cdot[E]$ in $H^{2}(Q, Z N)$. This group is useful in computing $|A u t(P)|$. In case $N, Q$ are elementary Abelian 2-groups our results involve the theory of quadratic forms and the Arf invariant.
\end{abstract}

\section{Introduction}

Since the simple modules of a ring and its semi-simple quotient are the same, for many purposes it suffices to consider the latter ring. In this note we study the problem of calculating the semi-simple quotient of the group ring $\mathbf{F}_{p} A u t(P)$ for the automorphism group of a finite $p$-group. The usual method is to consider the maximal elementary Abelian quotient $P \rightarrow P / \Phi(P)$, where $\Phi(P)$ is the Frattini subgroup. The induced map $A u t(P) \rightarrow A u t(P / \Phi(P))$ has a $p$-group as kernel by the Hall Basis Theorem. Hence the map of algebras

$$
\mathbf{F}_{p} A u t(P) \rightarrow \mathbf{F}_{p} A u t(P / \Phi(P))
$$

has a nilpotent kernel and thus suffices to compute the semi-simple quotient. However this map is not necessarily onto and one is left with the still considerable problem of determining the image. For $\Phi(P)=\mathbf{Z} / p$, when $p$ is an odd prime, this has been done by Dietz [7] giving a complete determination of $A u t(P)$.

We adopt an inductive approach via extensions; that is, we assume $P$ is given as an extension

$$
E: N \rightarrow P \rightarrow Q
$$

with $\operatorname{Aut}(N), \operatorname{Aut}(Q)$ under control. Then there is an exact sequence relating the automorphism groups of $N$ and $Q$ with that of $P$, depending on the cohomology class of the extension $[E] \in H^{2}(Q, Z(N))$ where $Z(N)$ denotes the center of $N$.

Received October 29, 2002, revised February 28, 2003; published on March 14, 2003. 2000 Mathematics Subject Classification: Primary 20J06; Secondary 55P42

Key words and phrases: automorphism group, extension class, semi-simple quotient, stable splittings.

(c) 2003, John Martino and Stewart Priddy. Permission to copy for private use granted. 
Our motivation comes from stable homotopy theory. Let $G$ be a finite group and $p$ be a prime. The classifying space of $G$ completed at $p, B G_{p}^{\wedge}$, decomposes stably into a wedge of indecomposable summands

$$
B G_{p}^{\wedge} \simeq X_{1} \vee X_{2} \vee \cdots \vee X_{n} .
$$

Each summand $X_{i}$ is the mapping telescope of a primitive idempotent $e$ in the ring of stable self-maps of $B G_{p}^{\wedge}, e \in\left\{B G_{p}^{\wedge}, B G_{p}^{\wedge}\right\}$. Thus there is a one-to-one correspondence between the indecomposable summands and the simple modules of the ring of stable self-maps. This correspondence is explored in both $[\mathbf{2}]$ and [9] (see also $[\mathbf{1 0}])$. It turns out that modular representation theory plays a crucial role: if $P$ is a Sylow $p$-subgroup of $G$ then each indecomposable summand of $B G_{p}^{\wedge}$ originates in $B Q$ for some subgroup $Q \leqslant P$ and corresponds to a simple $\mathbf{F}_{p} A u t(Q)$ module.

Of course, the automorphism group of a group is of intrinsic interest in its own right, and our methods shed some light on its structure.

An outline of the paper follows: Section 2 covers the preliminaries on extensions, $E: N \rightarrow G \rightarrow Q$, including the fundamental exact sequence, Theorem 2.1, relating $\operatorname{Aut}(N), \operatorname{Aut}(G), \operatorname{Aut}(Q)$, and the extension class $[E]$. In Section 3 we define and identify a group structure, $\Omega([E])$, on the intersection of the two orbits $\operatorname{Aut}(N) \cdot[E]$ and $\operatorname{Aut}(Q) \cdot[E]$ where $\operatorname{Aut}(N)$ and $\operatorname{Aut}(Q)$ act on $H^{2}(Q, Z(N))$ in the usual way. This group, which we call the intersection orbit group, is useful in computing $|A u t(G)|$. The case of trivial action (or twisting) of $Q$ on $Z(N)$ is considered in Section 4. Extensions with $N, Q$, elementary Abelian $p$-groups are studied in Section 5 . In case $p=2$ this involves the theory of quadratic forms over $\mathbf{F}_{2}$ and the Arf invariant. We recall Browder's classification theorem $[\mathbf{3}]$ and give several results describing the order of a quotient of $\operatorname{Aut}(G)$ by a normal $p$-subgroup in Section 6 . For more complicated $p$-groups $G$ we describe an inductive procedure for extending these results using the mod- $p$ lower central series. Section 7 is devoted to several applications of the theory.

In what follows all groups are assumed finite, except as noted in Section 3.

\section{Preliminaries}

We begin by recalling the results of C. Wells [14] as extended by J. Buckley [4]. Because their notation is now non-standard, e.g., functions written on the right, we re-couch these results in more standard notation. Let

$$
E: N \stackrel{i}{\rightarrow} G \stackrel{\pi}{\rightarrow} Q
$$

be an extension of the group $N$ by the group $Q$ and let $A u t_{N}(G)$ be the group of automorphisms of $G$ mapping $N$ to itself. The obvious homomorphism $\rho=\left(\rho_{Q}, \rho_{N}\right)$ : $\operatorname{Aut}_{N}(G) \rightarrow \operatorname{Aut}(Q) \times \operatorname{Aut}(N)$ provides a means of studying $A u t_{N}(G)$.

As usual two extensions $E_{1}, E_{2}$ are equivalent $E_{1} \sim E_{2}$ if there is an isomorphism $\alpha: G_{1} \rightarrow G_{2}$ restricting to the identity on $N$ and inducing the identity on $Q$. The set of such equivalent extensions is denoted $\mathcal{E}(Q, N)$. The twisting (or coupling) $\chi: Q \rightarrow$ $\operatorname{Out}(N)$ of $E$ is the homomorphism defined as usual by $\chi(q)(n)=i^{-1}\left(g^{-1} i(n) g\right)$ where $g \in \pi^{-1}(q), n \in N$. Equivalent extensions have the same twisting. 
The center $Z N$ of $N$ has the structure of a $Q$ module via a homomorphism $\bar{\chi}: Q \rightarrow \operatorname{Aut}(Z N)$ defined by the composite

$$
\bar{\chi}: Q \stackrel{\chi}{\rightarrow} \operatorname{Out}(N) \stackrel{\text { res }}{\rightarrow} \operatorname{Aut}(Z N)
$$

where res $: \operatorname{Out}(N) \rightarrow \operatorname{Aut}(Z N)$ is induced by $\operatorname{Aut}(N) \rightarrow \operatorname{Aut}(Z N)$. It is wellknown that we may identify

$$
\mathcal{E}(Q, N)=\coprod_{\chi} H_{\bar{\chi}}^{2}(Q, Z N)
$$

where $\chi$ ranges over all twistings $\{Q \rightarrow O u t(N)\}$.

Now consider $(\sigma, \tau) \in \operatorname{Aut}(Q) \times \operatorname{Aut}(N)$ and form the extension

$$
\sigma E \tau^{-1}: N \stackrel{i \tau^{-1}}{\rightarrow} G \stackrel{\sigma \pi}{\rightarrow} Q
$$

Then $\operatorname{Aut}(Q) \times \operatorname{Aut}(N)$ acts on $\mathcal{E}(Q, N)$ from the left by

$$
(\sigma, \tau)[E]=\left[\sigma E \tau^{-1}\right] .
$$

One checks $(\sigma, \tau)\left(\sigma^{\prime}, \tau^{\prime}\right)[E]=\left(\sigma \sigma^{\prime}, \tau \tau^{\prime}\right)[E]$ and $(1,1)[E]=[E]$. The twisting of $(\sigma, \tau) E$ is given by $\gamma_{\bar{\tau}} \chi \sigma^{-1}$ where $\gamma_{\bar{\tau}}$ denotes conjugation by $\bar{\tau}$, the image of $\tau$ in $\operatorname{Out}(N)$. For a given $\chi$ define the subgroup $C_{\chi} \subset \operatorname{Aut}(Q) \times \operatorname{Aut}(N)$ by

$$
C_{\chi}=\left\{(\sigma, \tau) \in \operatorname{Aut}(Q) \times \operatorname{Aut}(N) \mid \gamma_{\bar{\tau}} \chi \sigma^{-1}=\chi\right\}
$$

that is, the following diagram commutes

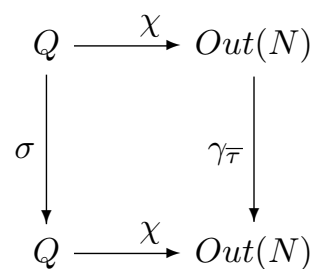

The subgroup $C_{\chi}$ consists of all ordered pairs $(\sigma, \tau) \in \operatorname{Aut}(Q) \times \operatorname{Aut}(N)$ that preserve the twisting.

If $\chi$ is trivial then clearly $C_{\chi}=\operatorname{Aut}(Q) \times \operatorname{Aut}(N)$. We note that $\operatorname{ker}(\chi)$ plays no role in the commutativity of the diagram and $\left.\sigma\right|_{\operatorname{ker}(\chi)}: \operatorname{ker}(\chi) \rightarrow \operatorname{ker}(\chi)$. Thus if the sequence

$$
\operatorname{ker}(\chi) \rightarrow Q \rightarrow i m(\chi)
$$

splits, e.g., if $Q$ is elementary Abelian, then $\left.\sigma\right|_{k e r(\chi)}$ can be an arbitrary linear isomorphism.

Then $C_{\chi}$ acts on $\{[E] \mid E$ has twisting $\chi\}$. It is trivial to check that $\operatorname{Im}(\rho) \subset C_{\chi}$ so we consider $\rho$ as a homomorphism $\rho: \operatorname{Aut}_{N}(G) \rightarrow C_{\chi}$.

Let $Z \frac{1}{\bar{\chi}}(Q, Z N)$ denote the group of derivations, i.e., functions $f: Q \rightarrow Z N$ satisfying $f\left(q q^{\prime}\right)=f(q)+q f\left(q^{\prime}\right)$ for $q, q^{\prime} \in Q$. Then there is a homomorphism $\mu: Z \frac{1}{\chi}(Q, Z N) \rightarrow A_{u t}(G)$ defined by $\mu(f)(g)=f(\pi(g)) \cdot g$.

Finally we define a function $\epsilon: C_{\chi} \rightarrow H_{\bar{\chi}}^{2}(Q, Z N)$ by restricting the action of $\operatorname{Aut}(Q) \times \operatorname{Aut}(N)$ on the extension class $[E]$, that is, $(\sigma, \tau) \mapsto(\sigma, \tau)[E]$. In general $\epsilon$ is not a homomorphism. 
The following is the principal result of $[\mathbf{1 4}]$ as extended by [4]:

Theorem 2.1. For a given extension $E: N \rightarrow G \rightarrow Q$ with twisting $\chi: Q \rightarrow \operatorname{Out}(N)$ there is an exact sequence

$$
1 \rightarrow Z_{\bar{\chi}}^{1}(Q, Z N) \stackrel{\mu}{\rightarrow} A u t_{N}(G) \stackrel{\rho}{\rightarrow} C_{\chi} \stackrel{\epsilon}{\rightarrow} H_{\bar{\chi}}^{2}(Q, Z N)
$$

with $\operatorname{Im}(\rho)=\left(C_{\chi}\right)_{[E]}$, the isotropy subgroup of $C_{\chi}$ fixing $[E]$. The map $\epsilon$ is not onto and is only a set map.

An alternate exact sequence results by replacing $Z_{\bar{\chi}}(Q, Z N)$ with the cohomology group $H_{\bar{\chi}}(Q, Z N)$ and $A u t_{N}(G)$ by $A u t_{N}(G) / \operatorname{Inn}_{Z N}(G)$ where $\operatorname{Inn}_{Z N}(G)$ is group of inner automorphisms of $G$ induced by elements of $Z N$, (see [12], Proposition IV 2.1). We shall be interested in $\operatorname{Im}(\rho)$ so we will not need this refinement.

In case $Z N$ is a $p$-group Theorem 2.1 shows $Z \frac{1}{\chi}(Q, Z N)$ is a normal $p$-subgroup of $A_{u t}(G)$ which in turn implies $\mathbf{F}_{p}\left(\left(C_{\chi}\right)_{[E]}\right)$ suffices to compute simple modules and idempotents:

Corollary 2.2. If $E: N \rightarrow G \rightarrow Q$ is an extension with $Z N$ a p-group, then $\mathbf{F}_{p} A u t_{N}(G) \stackrel{\mathbf{F}_{p}(\rho)}{\longrightarrow} \mathbf{F}_{p}\left(\left(C_{\chi}\right)_{[E]}\right)$ is surjective with nilpotent kernel.

Proof. Since $\operatorname{ker}(\rho)=Z_{\bar{\chi}}(Q, Z N)$ is a $p$-group it is well known that $\operatorname{ker}\left(\mathbf{F}_{p}(\rho)\right)$ is nilpotent.

We shall be interested in determining when $A_{u t}(G)$ is a $p$-group; clearly this is true if $\operatorname{Aut}(Q)$ and $\operatorname{Aut}(N)$ are $p$-groups. Further

Corollary 2.3. Suppose $G$ is a p-group with $p$ odd, and $N$ is generated by the elements of $G$ of order $p$. Then $\operatorname{Aut}(G)$ is a p-group if Aut $(N)$ is a p-group.

Proof. By hypothesis $N=\Omega_{1}(G)$ is a characteristic subgroup, hence $A u t_{N}(G)=$ $\operatorname{Aut}(G)$. If $\alpha \in \operatorname{Aut}(G)$ has $p^{\prime}$ order then $\rho_{N}(\alpha)=i d_{N}$. Since $p$ is odd, the automorphisms of $G$ which have $p^{\prime}$ order and fix $N$ are trivial by Theorem 5.3.10, [8]. Thus $\operatorname{Aut}(G)$ is a $p$-group.

\section{The Intersection Orbit Group}

Let $X$ be a left $A \times B$ set where $A, B$ are groups, not necessarily finite. Equivalently $A$ acts on the left, $B$ acts on the right such that $a(x b)=(a x) b$, and $(a, b) x=a x b^{-1}$, where $a \in A, x \in X, b \in B$. As usual let $A x$ denote the orbit of $x$ under the action of $A$ (respectively $x B$ denote the orbit of $x$ under the action of $B$ ). We define the intersection orbit group at $x$

$$
\Omega(x):=(A x) \cap(x B)
$$

If $a x=x b, a^{\prime} x=x b^{\prime}$ are elements of $\Omega(x)$, their product is defined by

$$
(a x)\left(a^{\prime} x\right):=\left(a a^{\prime}\right) x=x\left(b b^{\prime}\right)
$$

It is straightforward to check that this pairing is well-defined giving $\Omega(x)$ the structure of a group. Although left and right actions commute, $\Omega(x)$ is not necessarily 
Abelian; however, if either $A$ or $B$ is a $p$-group, then $\Omega(x)$ is a $p$-group. Also if $A$ or $B$ is trivial then obviously $\Omega(x)=\{x\}$, the trivial group.

Again, as usual, let $A_{x}$ and $B_{x}$ denote the respective isotropy subgroups.

Proposition 3.1. There is an isomorphism of groups

$$
\phi:(A \times B)_{x} /\left(A_{x} \times B_{x}\right) \stackrel{\cong}{\longrightarrow} \Omega(x)
$$

given by $\phi(a, b)=a x=x b^{-1}$.

Proof. $(a, b) x=x$ if and only if $a x=x b^{-1}$. Similarly $(a, b) \in A_{x} \times B_{x}$ if and only if $a x=x b^{-1}=x$. Thus $\phi$ is well-defined and bijective. It is a homomorphism by the definition of the product in $\Omega(x)$.

Corollary 3.2. If $A$ and $B$ are finite groups, then

$|\Omega(x)|$ divides

$$
\left|N_{A}\left(A_{x}\right) / A_{x}\right|,\left|N_{B}\left(B_{x}\right) / B_{x}\right|,
$$

and

$$
\operatorname{gcd}(|A x|,|x B|) .
$$

Proof. Note that $N_{A}\left(A_{x}\right) / A_{x}$ is the largest subset of $A x \cong A / A_{x}$ which is a group. Thus $\Omega(x) \leqslant N_{A}\left(A_{x}\right) / A_{x}$ is a subgroup and so $|\Omega(x)|$ divides $\left|N_{A}\left(A_{x}\right) / A_{x}\right|$ and hence $A / A_{x}$. Similarly for $B$. Thus $|\Omega(x)|$ divides $\operatorname{gcd}(|A x|,|x B|)$.

Returning to automorphism groups, the following result often simplifies computing $|\operatorname{Im}(\rho)|$.

Corollary 3.3. For a given extension of finite groups $E: N \rightarrow G \rightarrow Q$,

i) $|\operatorname{Im}(\rho)|=\left|\operatorname{Aut}(Q)_{[E]}\right| \cdot\left|\operatorname{Aut}(N)_{[E]}\right| \cdot|\Omega([E])|$

ii) $\operatorname{Im}(\rho)$ is a p-group if and only if $\operatorname{Aut}(Q)_{[E]}, \operatorname{Aut}(N)_{[E]}$, and $|\Omega([E])|$ are p-groups.

Proof. ii) follows from i) which follows immediately from Proposition 3.1 with $A=$ $\operatorname{Aut}(Q), B=\operatorname{Aut}(N)$.

There exists extensions that do not depend on the intersection orbit group.

Proposition 3.4. Suppose $N$ is an Abelian p-group, Aut $(Q)$ is a p-group, and $H^{2}(Q, N) \neq 0$ with a trivial twisting. Then there exists a non-split extension $[E]$ such that

$$
\left|A u t_{N}(G)\right|_{p}=|\operatorname{Hom}(Q, N)||A u t(Q)||A u t(N)|_{p}
$$

Proof. By Corollary 3.2, it suffices find $[E]$ with $|\Omega([E])|=1$. Let $A u t(Q) \times S$ be a Sylow $p$-subgroup of $A u t(Q) \times \operatorname{Aut}(N)$. The $\operatorname{Aut}(Q) \times A u t(N)$-module $H^{2}(Q, N)$ has cardinality a power of $p$. Thus there is an $A u t(Q) \times S$ fixed point, $[E] \neq 0$ by the fixed point result $[\mathbf{1 3}]$, p. 64, which generalizes to this case). Thus $\operatorname{Aut}(Q)_{[E]}=\{[E]\}$ and $|S|=|\operatorname{Aut}(N)|_{p}$. The kernel of the exact sequence in Theorem 2.1 is $Z^{1}(Q, N)=$ $\operatorname{Hom}(Q, N)$, which explains the presence of that term in the proposition. 


\section{Trivial twisting}

Proposition 4.1. If $E: N \rightarrow G \rightarrow Q$ has trivial twisting the exact sequence of Theorem 2.1 reduces to

$$
0 \rightarrow \operatorname{Hom}(Q, Z N) \stackrel{\mu}{\rightarrow} \operatorname{Aut}_{N}(G) \stackrel{\rho}{\rightarrow} \operatorname{Aut}(Q) \times \operatorname{Aut}(N) \rightarrow H^{2}(Q, Z N)
$$

Proof. [4], Th. 3.1. Clearly $Z_{\bar{\chi}}(Q, Z N)=\operatorname{Hom}(Q, Z N)$ and $C_{\chi}=\operatorname{Aut}(Q) \times$ $\operatorname{Aut}(N)$.

Before proceeding, it is instructive to consider the simplest case $G=N \times Q$. The extension class is trivial thus $\rho: \operatorname{Aut}_{N}(G) \rightarrow \operatorname{Aut}(Q) \times \operatorname{Aut}(N)$ is surjective. In fact $\rho$ is split by the usual inclusion $\operatorname{Aut}(Q) \times \operatorname{Aut}(N) \rightarrow A u t_{N}(G)$. For a more complete discussion of $\operatorname{Aut}(Q \times N)$ see [11].

In what follows we are interested in $G=P$, a $p$-group. As immediate corollaries of 4.1 we have

Corollary 4.2. If $E: N \rightarrow P \rightarrow Q$ has trivial twisting and Aut $(N)$ and $\operatorname{Aut}(Q)$ both p-groups then $\operatorname{Aut}_{N}(P)$ is a p-group.

Corollary 4.3. Let $P$ be a p-group and define $P_{1}=P, P_{i+1}=P_{i} / Z\left(P_{i}\right)$. Suppose Aut $\left(Z\left(P_{i}\right)\right)$ is a p-group for each $i$. Then $\operatorname{Aut}(P)$ is a p-group. In particular this hypothesis holds if $p=2$ and for each $i$ the summands of $P_{i}$ have distinct exponents.

Proof. The first statement is clear from Corollary 4.2. Similarly the second follows from Proposition 4.5 of $[\mathbf{1 1}]$ which implies each $A u t\left(P_{i}\right)$ is a 2-group.

Another particularly tractable case is that of $E: N \rightarrow P \rightarrow Q$, with $N$ an Abelian, characteristic subgroup. Since $\operatorname{Hom}(Q, N)$ tends to be relatively large in this case, the quotient $\operatorname{Im}(\rho)$ can be significantly smaller than $\operatorname{Aut}(G)$. We examine this phenomenon in more detail.

Let $\left\{\Gamma^{n}(P)\right\}$ be the mod-p lower central series, that is $\Gamma^{0}(P)=P$ and

$$
\Gamma^{n}(P)=\left\langle\left(g_{1}, \ldots, g_{s}\right)^{p^{k}} \mid s p^{k}>n\right\rangle, \quad n \geqslant 1
$$

where $\left(g_{1}, \ldots, g_{s}\right)=\left(g_{1},\left(g_{2},\left(\ldots\left(g_{s-1}, g_{s}\right) \ldots\right)\right)\right.$ is the $s$-fold iterated commutator. Then $\Gamma^{1}(P)=\Phi(P)$ is the Frattini subgroup and $V=P / \Gamma^{1}(P)$ is the largest elementary Abelian quotient of $P$. For some $n, \Gamma^{n}(P)=1$.

Example 4.4. For $p$ an odd prime we consider the group

$P=\langle a, b, c| a^{p^{2}}=b^{p^{2}}=c^{p}=1, c=(b, a)$, trivial higher commutators $\rangle$

We shall determine $\operatorname{Aut}\left(P / \Gamma^{p}(P)\right)$ using the extension

$$
E: N=\Gamma^{1}(P) / \Gamma^{p}(P) \rightarrow P / \Gamma^{p}(P) \rightarrow Q=P / \Gamma^{1}(P)
$$

where $N=\mathbf{Z} / p\left\langle a^{p}, b^{p}, c\right\rangle$ and $Q=\mathbf{Z} / p\langle\bar{a}, \bar{b}\rangle$ are elementary Abelian. This extension has trivial twisting and its extension cocycle is easily seen to be $[E]=(x \wedge y) \otimes c \in$ $H^{2}(V, Q)=H^{2}(V) \otimes \mathbf{Z} / p\langle z\rangle$ where $x, y$ are dual to $a, b$ respectively. By inspection

$$
\operatorname{Im}(\rho)=\left\langle(A, B) \in G L_{2}\left(\mathbf{F}_{p}\right) \times G L_{1}\left(\mathbf{F}_{p}\right) \mid B=\operatorname{det}(A)\right\rangle .
$$

Thus $\operatorname{Im}(\rho) \cong G L_{2}\left(\mathbf{F}_{p}\right)$. We conclude $\mathbf{F}_{p} \operatorname{Aut}(P) \rightarrow \mathbf{F}_{p} G L_{2}\left(\mathbf{F}_{p}\right)$ is surjective with nilpotent kernel. Finally we note $\operatorname{Hom}(Q, N)=\operatorname{Mat}_{3,2}\left(\mathbf{F}_{p}\right)$, thus $|\operatorname{Hom}(Q, N)|=$ $p^{6}$ and $|A u t(P)|=p^{6}\left|G L_{2}\left(\mathbf{F}_{p}\right)\right|=p^{7}\left(p^{2}-1\right)(p-1)$. 
Example 4.5. Let $U_{5}\left(\mathbf{F}_{2}\right) \leqslant G L_{5}\left(\mathbf{F}_{2}\right)$ be the unipotent subgroup of upper triangular matrices over $\mathbf{F}_{2}$. These matrices necessarily have ones on the diagonal. Thus $U_{5}\left(\mathbf{F}_{2}\right)$ is generated by $x_{i j}=I_{4}+e_{i j}$ where $1 \leqslant i<j \leqslant 5$ and $e_{i j}$ is the standard elementary matrix with 1 in the $i j$ position and zeros elsewhere. Let $P=U_{5}\left(\mathbf{F}_{2}\right) / \Gamma^{2} U_{5}\left(\mathbf{F}_{2}\right)$. Then there is a central extension

$$
E: N=\mathbf{Z} / 2\left\langle\bar{x}_{13}, \bar{x}_{24}, \bar{x}_{35}\right\rangle \rightarrow P \rightarrow Q=\mathbf{Z} / 2\left\langle\bar{x}_{12}, \bar{x}_{23}, \bar{x}_{34}, \bar{x}_{45}\right\rangle
$$

Since $N$ is the commutator subgroup of $P$, it is characteristic. The extension cocycle is

$$
[E]=y_{12} y_{23} \otimes \bar{x}_{13}+y_{23} y_{34} \otimes \bar{x}_{24}+y_{34} y_{45} \otimes \bar{x}_{35}
$$

where

$$
H^{*}(Q, N)=H^{*}(Q) \otimes N=\mathbf{Z} / 2\left\langle y_{12}, y_{23}, y_{34}, y_{45}\right\rangle \otimes\left\langle\bar{x}_{13}, \bar{x}_{24}, \bar{x}_{35}\right\rangle
$$

and $y_{i j}$ is dual to $\bar{x}_{i j}$. Since the twisting is trivial, $C_{\chi}=\operatorname{Aut}(Q) \times \operatorname{Aut}(N)=$ $G L_{4}\left(\mathbf{F}_{2}\right) \times G L_{3}\left(\mathbf{F}_{2}\right)$. Direct calculation shows

$$
(\operatorname{Aut}(Q) \times \operatorname{Aut}(N))_{[E]}=\left\langle A, B \mid A^{4}=B^{2}=1, B A B=A^{-1}\right\rangle
$$

the dihedral group of order 8 generated by

$$
\begin{aligned}
A & =\left(\begin{array}{llll}
0 & 0 & 0 & 1 \\
0 & 0 & 1 & 0 \\
0 & 1 & 0 & 0 \\
1 & 0 & 1 & 0
\end{array}\right) \times\left(\begin{array}{lll}
0 & 0 & 1 \\
1 & 1 & 0 \\
1 & 0 & 0
\end{array}\right) \\
B & =\left(\begin{array}{llll}
1 & 0 & 0 & 0 \\
0 & 1 & 0 & 0 \\
0 & 0 & 1 & 0 \\
0 & 1 & 0 & 1
\end{array}\right) \times\left(\begin{array}{lll}
1 & 0 & 0 \\
0 & 1 & 1 \\
0 & 0 & 1
\end{array}\right)
\end{aligned}
$$

Moreover $|\operatorname{Hom}(Q, N)|=2^{12}, \operatorname{Aut}(P)$ is a 2 group of order $2^{15}$.

\section{An inductive procedure for determining $\operatorname{Aut}(P)$}

Let $G=P$ be a $p$-group. Since $\Gamma^{i}(P)$ is characteristic there is an induced homomorphism $\rho_{V}: \operatorname{Aut}(P) \rightarrow \operatorname{Aut}(V)$ which factors as

$$
\begin{aligned}
\rho_{V}: \operatorname{Aut}(P) & \rightarrow \cdots \rightarrow \operatorname{Aut}\left(P / \Gamma^{i+1}(P)\right) \stackrel{\rho_{i}}{\rightarrow} \operatorname{Aut}\left(P / \Gamma^{i}(P)\right) \\
& \rightarrow \cdots \stackrel{\rho_{2}}{\rightarrow} \operatorname{Aut}\left(P / \Gamma^{2}(P)\right) \stackrel{\rho_{1}}{\rightarrow} \operatorname{Aut}(V)
\end{aligned}
$$

We shall describe an inductive procedure for lifting elements in the image of this map.

Consider the extensions

$$
\begin{gathered}
E_{i}: \Gamma^{1}(P) / \Gamma^{i}(P) \rightarrow P / \Gamma^{i}(P) \rightarrow V, \quad i \geqslant 2 \\
\widetilde{E}_{i}: \Gamma^{i}(P) / \Gamma^{i+1}(P) \rightarrow \Gamma^{1}(P) / \Gamma^{i+1}(P) \rightarrow \Gamma^{1}(P) / \Gamma^{i}(P), \quad i \geqslant 2 .
\end{gathered}
$$


where $E_{2}$ and $\widetilde{E}_{i}$ have trivial twisting. In each case the kernel is a characteristic subgroup.

Let $\sigma_{1} \in \operatorname{Aut}(V)$. By Theorem 2.1,

$$
\sigma_{1} \in \operatorname{Im}\left\{\operatorname{Aut}_{\Gamma^{1}(P) / \Gamma^{2}(P)}\left(P / \Gamma^{2}(P)\right) \rightarrow \operatorname{Aut}(V)\right\}
$$

if and only if there exist $\tau_{1} \in \operatorname{Aut}\left(\Gamma^{1}(P) / \Gamma^{2}(P)\right)$ such that $\left(\sigma_{1}, \tau_{1}\right)$ fixes

$$
\left[E_{2}\right] \in H^{2}\left(V, \Gamma^{1}(P) / \Gamma^{2}(P)\right) .
$$

Then there exists $\sigma_{2} \in A u t_{\Gamma^{1}(P) / \Gamma^{2}(P)}\left(P / \Gamma^{2}(P)\right)$ lifting $\sigma_{1}$. Since

$$
\operatorname{Aut}_{\Gamma^{1}(P) / \Gamma^{2}(P)}\left(P / \Gamma^{2}(P)\right)=\operatorname{Aut}\left(P / \Gamma^{2}(P)\right)
$$

this completes the initial step. Now suppose inductively that we have found elements $\sigma_{i} \in \operatorname{Aut}\left(P / \Gamma^{i}(P)\right), \tau_{i-1} \in \operatorname{Aut}\left(\Gamma^{1}(P) / \Gamma^{i}(P)\right)$ such that $\sigma_{i}, \tau_{i-1}$ are lifts of $\sigma_{1}, \tau_{i-2}$, respectively. We need to find $\tau_{i} \in \operatorname{Aut}\left(\Gamma^{1}(P) / \Gamma^{i+1}(P)\right)$ such that $\left(\sigma_{1}, \tau_{i}\right) \in C_{\chi}$ fixes

$$
\left[E_{i+1}\right] \in H_{\bar{\chi}}^{2}\left(V, Z\left(\Gamma^{1}(P) / \Gamma^{i+1}(P)\right)\right) .
$$

Then by Thm 2.1 there exists $\sigma_{i+1} \in \operatorname{Aut}\left(P / \Gamma^{i}(P)\right)$ lifting $\sigma_{1}$.

To find $\tau_{i}$ we apply the same technique to the extension $\widetilde{E}_{i}$ noting that the twisting is trivial so condition (1) is trivially satisfied. Thus we must find $\tau_{i}^{\prime} \in$ $\operatorname{Aut}\left(\Gamma^{i}(P) / \Gamma^{i+1}(P)\right)$ such that $\left(\tau_{i-1}, \tau_{i}^{\prime}\right)$ fixes

$$
\left[\widetilde{E}_{i}\right] \in H^{2}\left(\Gamma^{1}(P) / \Gamma^{i}(P), \Gamma^{i}(P) / \Gamma^{i+1}(P)\right)
$$

since $\Gamma^{i}(P) / \Gamma^{i+1}(P)$ is its own center. Then applying Theorem 2.1 again there exists $\tau_{i} \in \operatorname{Aut}\left(\Gamma^{1}(P) / \Gamma^{i+1}(P)\right)$ lifting $\tau_{i-1}$ as desired.

The induction terminates when $\Gamma^{i+1}(P)=1$.

The procedure described in this section is demonstrated in the examples in Section 7 .

\section{Extensions of elementary Abelian groups}

We consider extensions $E: N \rightarrow G \rightarrow V$ where $N, V$ are elementary Abelian $p$ groups with $N$ central. In this case the twisting is trivial, $\chi=i d$ and $C_{\chi}=\operatorname{Aut}(N) \times$ $\operatorname{Aut}(V)$. Our aim is to use Corollary 3.3 to compute $|\operatorname{Im}(\rho)|$. Let $n=\operatorname{dim}_{\mathbf{F}_{p}}(N)$. Then the extension cocycle $[E] \in H^{2}(V ; N)$ has the form $[E]=\left(X_{1}, X_{2}, \ldots, X_{n}\right)$ where $X_{i} \in H^{2}\left(V ; \mathbf{F}_{p}\right)$. We recall that $\operatorname{Aut}(V)$ acts diagonally on $[E], \sigma[E]=$ $\left(\sigma X_{1}, \sigma X_{2}, \ldots, \sigma X_{n}\right)$ for $\sigma \in \operatorname{Aut}(V)$. Thus the isotropy subgroup

$$
\operatorname{Aut}(V)_{[E]}=\operatorname{Aut}(V)_{X_{1}} \cap \cdots \cap \operatorname{Aut}(V)_{X_{n}}
$$

The action of $\operatorname{Aut}(N)$ on $[E]$ is induced from that on $N$.

\subsection{Quadratic Forms}

At this point we restrict our attention to the case $p=2$. Let $m=\operatorname{dim}(V)$ then each $X_{i}$ is a quadratic form in $x_{1}, x_{2}, \ldots, x_{m}$ the generators of $H^{*}\left(V ; \mathbf{F}_{2}\right)=$ $\mathbf{F}_{2}\left[x_{1}, x_{2}, \ldots, x_{m}\right],\left|x_{i}\right|=1$. 
We recall some classical facts about quadratic forms $Q: V \rightarrow \mathbf{F}_{2},[\mathbf{3}],[\mathbf{6}],[\mathbf{5}]$. The defining property is that the associated form $B(x, y)=Q(x+y)+Q(x)+Q(y)$ is alternate bilinear.

The bilinear radical of $B$,

$$
\operatorname{bilrad}(V, B):=\{x \in V \mid B(x, y)=0, \forall y \in V\}
$$

As usual, $B$ is called non-degenerate if $\operatorname{bilrad}(V, B)=0$, i.e. its matrix is nonsingular. The radical of $Q$,

$$
\operatorname{Rad}(V, Q):=\{x \in \operatorname{bilrad}(V, B) \mid Q(x)=0\}
$$

$Q$ is said to be non-degenerate if $\operatorname{Rad}(V, Q)=0$.

By a theorem of Dickson [6] (Section 199) a (non-zero) quadratic form over $\mathbf{F}_{2}$ in $m$ variables which is not equivalent (by a change of basis) to one in fewer variables must be equivalent to one of the following standard non-degenerate quadratic forms

$$
\begin{array}{crl}
\Phi_{m}^{+} & =x_{1} x_{2}+\cdots+x_{m-1} x_{m}, & m \text { even } \\
\Phi_{m}^{-}=x_{1} x_{2}+\cdots+x_{m-3} x_{m-2}+x_{m-1}{ }^{2}+x_{m-1} x_{m}+x_{m}{ }^{2}, & m \text { even } \\
\Phi_{m}=x_{1}{ }^{2}+x_{2} x_{3}+\cdots+x_{m-1} x_{m}, & m \text { odd }
\end{array}
$$

If $\operatorname{bilrad}(V, B)=0$, then $m=2 r$ is even and one can define the Arf invariant of $Q$ with respect to a symplectic basis $\left\{u_{1}, v_{1}, \ldots, u_{k}, v_{k}\right\}$ by

$$
\operatorname{Arf}(Q)=\sum_{i=1}^{k} Q\left(u_{i}\right) Q\left(v_{i}\right) \in \mathbf{Z} / 2
$$

This is invariant of the choice of symplectic basis and determines $Q$ up to equivalence. It is convenient to write $\mathbf{Z} / 2=\langle \pm 1\rangle$ multiplicatively. Then with this notation, W. Browder has shown that $\operatorname{Arf}(Q)=1,-1$ if and only if $Q$ sends the majority of elements of $V$ to $1,-1$ respectively [3]. For $m$ even one finds $\operatorname{Arf}\left(\Phi_{m}^{+}\right)=1$, $\operatorname{Arf}\left(\Phi_{m}^{-}\right)=-1$.

The Arf invariant can be extended to the $m$ odd case (where $B$ is degenerate) as follows. It is easy to see that $\Phi_{m}$ sends the same number of elements to 1 and -1 thus one can define $\operatorname{Arf}\left(\Phi_{m}\right)=0$. It is clear that Browder's definition (also known as the "democratic invariant") is invariant under any basis change. This leads to the following classification theorem.

Theorem 6.1. [3], Theorem III.1.14

$A$ quadratic form $Q: V \rightarrow \mathbf{Z} / 2$ is determined up to equivalence by the triple $(\operatorname{dim}(V), \operatorname{dim}(\operatorname{bilrad}(V, B)), \operatorname{Arf}(Q))$

The action of $\operatorname{Aut}(N)=G L\left(n, \mathbf{F}_{2}\right)$ on an $n$-tuple $\left(X_{1}, X_{2}, \ldots, X_{n}\right)$ of quadratic forms is linear and thus involves the sum of forms. Unfortunately it is impossible, in general, to determine the sum from the Arf invariant. For example if $X_{1}=x^{2}$ and $X_{2}=x y+y^{2}$ then $\operatorname{Arf}\left(X_{1}+X_{2}\right) \neq \operatorname{Arf}\left(X_{1}\right)+\operatorname{Arf}\left(X_{2}\right)$. However, on a direct sum of vector spaces then it follows easily from the definition that

$$
\operatorname{Arf}\left(X_{1} \oplus X_{2}\right)=\operatorname{Arf}\left(X_{1}\right)+\operatorname{Arf}\left(X_{2}\right) .
$$


For the rest of this subsection we restrict attention to $m$ even and consider the special case $[E]=\left(X_{1}, X_{2}, \ldots, X_{n}\right)$ where the $X_{i}$ are in standard form. We write $X_{i}=X$ or $Y$ depending on whether the Arf invariant is 1 or -1 . In the following theorem we use $\sim$ to denote conjugacy.

Theorem 6.2. Suppose $m=2 r$ and $[E]=\left(X_{1}, X_{2}, \ldots, X_{n}\right)$ with $X_{1}=\cdots=$ $X_{k}=X, X_{k+1}=\cdots=X_{n}=Y$. Then $\Omega([E])=[E]$ and $|\operatorname{Im}(\rho)|=\left|\operatorname{Aut}(V)_{[E]}\right| \cdot$ $\mid$ Aut $(N)_{[E]} \mid$. Furthermore

1) If $k=n$ then

$$
\operatorname{Aut}(V)_{[E]}=O_{m}^{+}\left(\mathbf{F}_{2}\right)
$$

the orthogonal group of order $2\left(2^{r}-1\right) \prod_{i=1}^{r-1}\left(2^{2 i}-1\right) 2^{2 i}$ of matrices preserving the form $X$.

$$
\operatorname{Aut}(N)_{[E]} \sim\left(\begin{array}{cc}
1 & 0 \\
* & G L_{n-1}\left(\mathbf{F}_{2}\right)
\end{array}\right)
$$

of order $2^{n-1} \prod_{i=1}^{n-1}\left(2^{i}-1\right) 2^{i-1}$. Im $(\rho)$ is a 2-group if and only if $m, n<3$.

2) If $k=0$ then

$$
\operatorname{Aut}(V)_{[E]}=O_{m}^{-}\left(\mathbf{F}_{2}\right)
$$

the orthogonal group of order $2\left(2^{n}+1\right) \prod_{i=1}^{n-1}\left(2^{2 i}-1\right) 2^{2 i}$ of matrices preserving the form $Y$ and

$$
\operatorname{Aut}(N)_{[E]} \sim\left(\begin{array}{cc}
1 & 0 \\
* & G L_{n-1}\left(\mathbf{F}_{2}\right)
\end{array}\right) .
$$

$\operatorname{Im}(\rho)$ is a 2-group if and only if $m<2$ and $n<3$.

3) If $1<k<n$ then

$$
\operatorname{Aut}(V)_{[E]}=O_{m}^{+}\left(\mathbf{F}_{2}\right) \cap O_{m}^{-}\left(\mathbf{F}_{2}\right)
$$

and

$$
\operatorname{Aut}(N)_{[E]} \sim\left(\begin{array}{cc}
I_{2} & 0 \\
* & G L_{n-2}\left(\mathbf{F}_{2}\right)
\end{array}\right)
$$

of order $2^{2(n-2)} \prod_{i=1}^{n-2}\left(2^{i}-1\right) 2^{i-1}$. Im $(\rho)$ is a 2-group if and only if $m, n<4$.

Proof. The calculation of $A u t(V)_{[E]}$ follows from (3). The intersection orbit group $\Omega([E])=(\operatorname{Aut}(V) \cdot[E]) \cap(\operatorname{Aut}(N) \cdot[E])=\{[E]\}$. To see this suppose $\sigma[E]=[E] \tau^{-1}$ differs from $[E]=\left(X_{1}, X_{2}, \ldots, X_{n}\right)$ in the $i$-th coordinate $X_{i}=X$, say. Now $\sigma X=$ $\left([E] \tau^{-1}\right)_{i}=a X+b Y, a, b \in \mathbf{F}_{2}$. Thus $\sigma X=X+Y$ since $\sigma X \neq X$ and $Y$ has Arf invariant -1 . However $\sigma X=X+Y=x_{m-1}^{2}+x_{m}^{2}=\left(x_{m-1}+x_{m}\right)^{2}$ contradicting the fact that $X$ is not equivalent to a quadratic form in fewer than $m$ variables. Similarly if $X_{i}=Y$.

Since $X$ and $Y$ are linearly independent polynomials, $(X, \ldots, X, Y, \ldots, Y)$ is equivalent to $(X, Y, 0, \ldots, 0)$ by a change of basis. Thus the descriptions of $A u t(N)_{[E]}$ follow immediately. 


\subsection{Non-standard Forms}

We now turn to the more general case where the forms $X_{i}$ of $[E]=\left(X_{1}, X_{2}, \ldots, X_{n}\right)$ are not in standard form. One can still determine $|\operatorname{Im}(\rho)|$; we illustrate this by considering the case $n=2, m=3$. Thus we consider pairs of quadratic forms $(X, Y)$ in variables $x, y, z$ each equivalent to (but not necessarily equal to) one of the standard four forms:

$$
x^{2}+y z, \quad x y, \quad x^{2}+x y+y^{2}, \quad x^{2} .
$$

The respective isotropy groups, as subgroups of $G L_{3}\left(\mathbf{F}_{2}\right)$, are

$$
\operatorname{Aut}(V)_{x^{2}+y z}=O_{3}\left(\mathbf{F}_{2}\right) \cong G L_{2}\left(\mathbf{F}_{2}\right)
$$

non-Abelian of order 6;

$$
\operatorname{Aut}(V)_{x y}=\left(\begin{array}{cc}
\Sigma_{2} & 0 \\
* & 1
\end{array}\right)
$$

elementary Abelian of rank 3;

$$
\operatorname{Aut}(V)_{x^{2}+x y+y^{2}}=\left(\begin{array}{cc}
G L_{2} & 0 \\
* & 1
\end{array}\right)
$$

of order 24;

$$
\operatorname{Aut}(V)_{x^{2}}=\left(\begin{array}{cc}
1 & 0 \\
* & G L_{2}
\end{array}\right)
$$

of order 24 .

\subsubsection{Simultaneous Equivalence}

First we consider the case where $X$ and $Y$ are simultaneously equivalent to a standard form, i.e., there is an invertible linear transformation $A$ of $V$ such that $A^{-1} X A$ and $A^{-1} Y A$ are each in standard form.

Case $1, X=Y$ :

Then $\operatorname{Aut}(V)_{(X, X)}=\operatorname{Aut}(V)_{X}$. It is also easy to see $\operatorname{Aut}(N)_{(X, X)}=\Sigma_{2}$ and the intersection of orbits $\Omega([E])=\{(X, X)\}$ in this case.

a) $X$ is equivalent to $x y$ : As above the isotropy subgroup $\operatorname{Aut}(V)_{(X, X)}$ is elementary Abelian of rank 3 . The intersection of the orbits is just $(X, X)$ so the order of $\operatorname{Im}(\rho)=8 \cdot 2 \cdot 1=16$.

b) $X$ is equivalent to $x^{2}+x y+y^{2}: \operatorname{Aut}(V)_{(X, X)}$ is of order 24 . Thus $|\operatorname{Im}(\rho)|=$ $24 \cdot 2 \cdot 1=48$.

c) $X$ is equivalent to $x^{2}+y z: A u t(V)_{(X, X)}=O_{3}\left(F_{2}\right)$. Thus $|\operatorname{Im}(\rho)|=6 \cdot 2 \cdot 1=12$.

d) $X$ is equivalent to $x^{2}$ : As above $\operatorname{Aut}(V)_{(X, X)}$ is of order 24. Thus $|\operatorname{Im}(\rho)|=$ $24 \cdot 2 \cdot 1=48$.

Case $2, X \neq Y$ :

In this case $\operatorname{Aut}(N)_{(X, Y)}=1$. Further $\operatorname{Aut}(V)_{(X, Y)}=\operatorname{Aut}(V)_{X} \cap \operatorname{Aut}(V)_{Y}=$ $\operatorname{Aut}(V)_{(Y, X)}$. There are several possibilities; we give only four in detail since the rest follow the same general pattern. By considering the Arf invariant we see that $\Omega([E])=1$ except in the third example. 
1) $(X, Y)$ equivalent to $\left(x^{2}+y z, x^{2}+x y+y^{2}\right)$ : Then $\operatorname{Aut}(V)_{X} \cap \operatorname{Aut}(V)_{Y}$ is

$$
O_{3}\left(\mathbf{F}_{2}\right) \cap\left(\begin{array}{cc}
G L_{2} & 0 \\
* & 1
\end{array}\right)=\mathbf{Z} / 2\left\langle\left(\begin{array}{ccc}
1 & 1 & 0 \\
0 & 1 & 0 \\
0 & 1 & 1
\end{array}\right)\right\rangle .
$$

The intersection of orbits is $\{(X, Y)\}$ hence $|\operatorname{Im}(\rho)|=2 \cdot 1 \cdot 1=2$.

2) $(X, Y)$ equivalent to $\left(x y, x^{2}+x y+y^{2}\right)$ : Then $\operatorname{Aut}(V)_{X} \cap \operatorname{Aut}(V)_{Y}$ is

$$
\left(\begin{array}{cc}
\Sigma_{2} & 0 \\
* & 1
\end{array}\right) \cap\left(\begin{array}{cc}
G L_{2} & 0 \\
* & 1
\end{array}\right)=\left(\begin{array}{cc}
\Sigma_{2} & 0 \\
* & 1
\end{array}\right)
$$

i.e., dihedral of order 8 . The intersection of orbits is $\{(X, Y)\}$ thus $|\operatorname{Im}(\rho)|=8 \cdot 1 \cdot 1=$ 8.

Similarly for $\left(x y, y^{2}+y z+z^{2}\right)$ and $\left(x z, x^{2}+x y+y^{2}\right)$.

3) $(X, Y)$ equivalent to $\left(x y, x^{2}+y z\right)$ : Then $\operatorname{Aut}(V)_{X} \cap \operatorname{Aut}(V)_{Y}$ is trivial and $\Omega([E])=\{(X, Y),(X+Y, Y)\}=\mathbf{Z} / 2$. Thus $|\operatorname{Im}(\rho)|=2$.

4) $(X, Y)$ equivalent to $(x y, y z)$ : Then $\operatorname{Aut}(V)_{X} \cap \operatorname{Aut}(V)_{Y}$ is trivial. Direct calculation shows the intersection of the orbits has order 6 . Thus $|\operatorname{Im}(\rho)|=1 \cdot 1 \cdot 6=$ 6 .

Similarly for $(x z, y z),(x z, x y)$, and $(y z, x z)$.

\subsubsection{Non-simultaneous Equivalence}

By this we mean $X$ and $Y$ are not simultaneously equivalent to a pair of standard forms. Since $X \neq Y, \operatorname{Aut}(N)_{(X, Y)}=1$ in all cases.

To illustrate this phenomena the following table gives a complete computation of $|\operatorname{Im}(\rho)|$ in case $X$ and $Y$ are equivalent (non-simultaneously) to $x^{2}+y z$. Then $X$ may be assumed to be $x^{2}+y z$ and we list only the relevant $Y$ 's and the corresponding values of $|\operatorname{Im}(\rho)|=2,3,4$.

$$
|\operatorname{Im}(\rho)|
$$

\begin{tabular}{|c|c|c|}
\hline 2 & 3 & 4 \\
\hline$x^{2}+x z+y z$ & $x y+x z+y^{2}$ & $x^{2}+y z+z^{2}$ \\
$x^{2}+x y+x z+z^{2}$ & $x^{2}+x y+x z+y^{2}+y z$ & $x y+x z+y^{2}+y z+z^{2}$ \\
$x^{2}+x z+y z+z^{2}$ & $x z+y^{2}+y z$ & $x y+x z+y z$ \\
$x^{2}+x y+y^{2}+y z$ & $x y+x z+z^{2}$ & $x z+y^{2}+z^{2}$ \\
$x^{2}+x y+y z$ & $x^{2}+x y+y^{2}+z^{2}$ & $x^{2}+y^{2}+y z$ \\
$x^{2}+x y+x z+y^{2}$ & $x^{2}+x z+y^{2}+z^{2}$ & $x y+z^{2}$ \\
& $x^{2}+x y+z^{2}$ & $x y+y^{2}+z^{2}$ \\
& $x z+y^{2}+y z+z^{2}$ & $x^{2}+y^{2}+y z+z^{2}$ \\
& $x^{2}+x z+y^{2}$ & $x z+y^{2}$ \\
& $x^{2}+x y+x z+y z+z^{2}$ & \\
& $x y+y^{2}+y z+z^{2}$ & \\
& $x y+y z+z^{2}$ & \\
\hline
\end{tabular}

In more detail, if $|\operatorname{Im}(\rho)|=2$, then $\operatorname{Aut}(V)_{(X, Y)}=1$ and $\Omega([E])=\{(X, Y)$, $(Y, X)\}=\mathbf{Z} / 2$ If $|\operatorname{Im}(\rho)|=3$, then $\left|\operatorname{Aut}(V)_{(X, Y)}\right|=1$ and $\Omega([E])=\{(X, Y),(X+$ 
$Y, X),(Y, X+Y)\}=\mathbf{Z} / 3$. If $|\operatorname{Im}(\rho)|=4$ then $\left|\operatorname{Aut}(V)_{(X, Y)}\right|=2$ and $\Omega([E])=$ $\{(X, Y),(Y, X)\}=\mathbf{Z} / 2$.

As a final example we consider

$X=x y+y^{2}, Y=x^{2}+y^{2}+y z+z^{2}$. To analyze $\operatorname{Aut}(V)_{(X, Y)}$ we separately reduce $X, Y$ to standard forms $f_{1}, f_{2}$ respectively

$$
\sigma_{1} X=f_{1}, \quad \sigma_{2} Y=f_{2}
$$

with $\sigma_{i} \in \operatorname{Aut}(V)$. Let $\sigma=\sigma_{2} \sigma_{1}^{-1}$, then $\sigma^{-1} f_{2}=\sigma_{1} Y$ hence

$$
\operatorname{Aut}(V)_{\left(f_{1}, \sigma_{1} Y\right)}=\operatorname{Aut}(V)_{f_{1}} \cap \sigma^{-1}\left[\operatorname{Aut}(V)_{f_{2}}\right] \sigma
$$

This determines $\operatorname{Aut}(V)_{(X, Y)}$ up to conjugacy since $\operatorname{Aut}(V)_{(X, Y)}=$ $\sigma_{1}{ }^{-1}\left[\operatorname{Aut}(V)_{\left(f_{1}, \sigma_{1} Y\right)}\right] \sigma_{1}$. In this example $f_{1}=x y, f_{2}=x^{2}+y z$ with $\sigma_{1}: y \mapsto x+y$, $\sigma_{2}=x \mapsto x+y+z$. Then

$$
\operatorname{Aut}(V)_{\left(f_{1}, \sigma_{1} Y\right)}=A_{u t} \cap \sigma^{-1}\left[\operatorname{Aut}(V)_{x^{2}+y z}\right] \sigma=\mathbf{Z} / 2\left\langle\left(\begin{array}{ccc}
1 & 0 & 0 \\
0 & 1 & 0 \\
0 & 1 & 1
\end{array}\right)\right\rangle
$$

Further $\Omega([E])=\{(X, Y),(X, X+Y)\}=\mathbf{Z} / 2$ hence $|\operatorname{Im}(\rho)|=2 \cdot 1 \cdot 2=4$.

\section{Applications}

1. Let $P$ denote the extraspecial group of order $|P|=p^{2 n+1}$ and exponent $p>2$ defined by the central extension

$$
E: Z \rightarrow P \rightarrow V
$$

where $Z=\Phi(P)=\mathbf{Z} / p, V=P / \Phi(P)=(\mathbf{Z} / p)^{2 n}$. The twisting $\chi$ is trivial thus $C_{\chi}=\operatorname{Aut}(V) \times \operatorname{Aut}(Z)$. Complete information about the automorphism group of $P$ as well as all other extensions of elementary Abelian $p$-groups by $\mathbf{Z} / p$ is known $[\mathbf{1 5}],[\mathbf{7}]$. Here we apply our results to obtain a quick derivation of $\operatorname{Im}\{\operatorname{Aut}(P) \stackrel{\rho}{\rightarrow}$ $\operatorname{Aut}(V) \times \operatorname{Aut}(Z)\}$.

$P$ is generated by elements $x_{1}, x_{2}, \ldots, x_{2 n}, \zeta$ of order $p$ satisfying

$$
\begin{gathered}
{\left[\zeta, x_{i}\right]=1} \\
{\left[x_{2 i-1}, x_{2 i}\right]=\zeta} \\
{\left[x_{2 i-1}, x_{j}\right]=1, \quad j \neq 2 i} \\
{\left[x_{2 i}, x_{j}\right]=1, \quad j \neq 2 i-1}
\end{gathered}
$$

Thus $\Phi(P)=Z(P)=\langle\zeta\rangle=\mathbf{Z} / p$ and $V=\left\langle\bar{x}_{1}, \bar{x}_{2}, \ldots, \bar{x}_{2 n}\right\rangle$. It is immediate from these relations that the extension cocycle is

$$
[E]=B \otimes \zeta
$$

where $B=y_{1} y_{2}+\cdots+y_{2 n-1} y_{2 n}, y_{i} \in H^{1}(V)$ is dual to $\bar{x}_{i}$. Since $p$ is an odd prime, $y_{i} y_{j}=-y_{j} y_{i}$. Thus $B$ is exactly the skew-symmetric form for the symplectic group $S p\left(n, \mathbf{F}_{p}\right)$. We shall need a slightly more general version $G S p\left(2 n, \mathbf{F}_{p}\right)$, [5], 
the transformations which fix $B$ up to a scalar. It is easy to see that $G S p\left(2 n, \mathbf{F}_{p}\right)=$ $\langle\gamma\rangle \rtimes S p\left(2 n, \mathbf{F}_{p}\right)$ where $\gamma$ is the linear transformation

$$
\bar{x}_{2 i-1} \mapsto k \bar{x}_{2 i-1}, \quad \bar{x}_{2 i} \mapsto \bar{x}_{2 i}
$$

and $k$ is a generator of $\mathbf{F}_{p}{ }^{*}$.

Now considering $(\sigma, \tau) \in A u t(Z) \times A u t(V)$ acting on $E$ we find

$$
(\sigma, \tau)(E)=\left[\sum_{i=1}^{n}(\sigma)\left(y_{2 i-1}\right) \sigma\left(y_{2 i}\right)\right] \otimes \tau(\zeta)
$$

Thus $(\sigma, \tau)(E)=E$ if and only if $\sigma \in G S p\left(2 n, \mathbf{F}_{p}\right)$ and $\tau$ is multiplication by $\operatorname{det}(\sigma)^{-1}$. We conclude $\operatorname{Im}\{\operatorname{Aut}(P) \rightarrow \operatorname{Aut}(V) \times \operatorname{Aut}(Z)\} \cong G \operatorname{Sp}\left(2 n, \mathbf{F}_{p}\right)$.

2. Let $W(n)$ be the universal $W$-group $[\mathbf{1}]$ on $n$ generators defined as the central extension

$$
1 \rightarrow N=\Phi(W(n)) \rightarrow W(n) \rightarrow Q=(\mathbf{Z} / 2)^{n} \rightarrow 1
$$

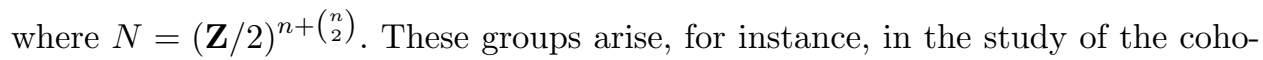
mology of Galois groups. The extension class $[E]=\left(X_{1}, X_{2}, \ldots, X_{n+\left(\begin{array}{c}n \\ 2\end{array}\right)}\right)$ where the $\left\{X_{i}\right\}$ form an ordered basis for $H^{2}(Q)=S^{2}\left(Q^{*}\right)$, the second symmetric power of the dual of $Q$. Any order will do. The twisting is trivial, hence $C_{\chi}=G L(Q) \times G L(N)$. Now $G L(Q)$ induces linear isomorphisms on $H^{*}(Q)$ which are determined by their values on squares in $H^{2}(Q)$. Thus given $\sigma \in G L(Q)$ we can find a $\tau \in G L(N)$ such that $(\sigma, \tau)([E])=(\sigma[E]) \tau^{-1}=[E]$. Thus we see that $\operatorname{Im}(\rho) \leqslant G L(Q) \times G L(N)$ by an injection which projects to an isomorphism on the first factor, i.e. $\operatorname{Im}(\rho) \cong$ $G L(Q)$.

3. We consider $\operatorname{Aut}(P)$ for the unipotent group $P=U_{4}\left(\mathbf{F}_{2}\right) \subset G L_{4}\left(\mathbf{F}_{2}\right)$ of upper triangular matrices over the finite field $\mathbf{F}_{2}$. These matrices necessarily have ones on the diagonal. Thus $P$ is generated by $\left\{x_{12}, x_{23}, x_{34}\right\}$. Then $\Gamma^{3}(P)=1, \Gamma^{2}(P)=$ $\mathbf{Z} / 2\left\langle x_{14}\right\rangle=Z P$, and $\Gamma^{1}(P)=\mathbf{Z} / 2\left\langle x_{13}, x_{24}, x_{14}\right\rangle$. The corresponding extensions have

$$
E_{2}: \Gamma^{1}(P) / \Gamma^{2}(P) \stackrel{i_{2}}{\rightarrow} P / \Gamma^{2}(P) \rightarrow V
$$

where $V=P / \Gamma^{1}(P)=\mathbf{Z} / 2\left\langle\bar{x}_{12}, \bar{x}_{23}, \bar{x}_{34}\right\rangle, \Gamma^{1}(P) / \Gamma^{2}(P)=\mathbf{Z} / 2\left\langle\bar{x}_{13}, \bar{x}_{24}\right\rangle$ and

$$
E_{3}: \Gamma^{1}(P) \stackrel{i_{3}}{\rightarrow} P \rightarrow V
$$

The twisting $\chi$ is trivial for $E_{2}$ but not for $E_{3}$. In this application we shall not need the auxiliary extensions $\left[\widetilde{E}_{i}\right]$.

Claim: $\operatorname{Im}\{\operatorname{Aut}(P) \rightarrow \operatorname{Aut}(V)\} \cong \Sigma_{3}$

Proof. (Sketch) From the commutator relations $\left[x_{i j}, x_{j k}\right]=x_{i k}$ for $i<j<k$, one sees that the extension class $\left[E_{2}\right] \in H^{2}\left(V ; \Gamma^{1}(P) / \Gamma^{2}(P)\right)$ is $x y \otimes \bar{x}_{13}+y z \otimes \bar{x}_{24}$ where

$$
\begin{gathered}
H^{*}\left(V, \Gamma^{1}(P) / \Gamma^{2}(P)\right)=H^{*}(V) \otimes \mathbf{Z} / 2\left\langle x_{13}, x_{24}\right\rangle \\
=\mathbf{Z} / 2[x, y, z] \otimes \mathbf{Z} / 2\left\langle x_{13}, x_{24}\right\rangle
\end{gathered}
$$


Here $x, y, z \in H^{1}(V)$ are classes dual to $\bar{x}_{12}, \bar{x}_{23}, \bar{x}_{34}$ respectively. Direct calculation shows the $\left[E_{2}\right]$ is fixed the subgroup generated by the involution defined by

$$
\sigma: \bar{x}_{12} \mapsto \bar{x}_{34}, \quad \bar{x}_{23} \mapsto \bar{x}_{23}, \quad \tau: x_{13} \mapsto x_{24}
$$

and the map of order three

$$
\begin{gathered}
\sigma^{\prime}: \bar{x}_{12} \mapsto \bar{x}_{12}+\bar{x}_{34}, \quad \bar{x}_{23} \mapsto \bar{x}_{23}, \quad \bar{x}_{34} \mapsto \bar{x}_{12}, \\
\tau^{\prime}: x_{13} \mapsto x_{24}, \quad x_{24} \mapsto x_{13}+x_{24}
\end{gathered}
$$

One finds $\left\langle(\sigma, \tau),\left(\sigma^{\prime}, \tau^{\prime}\right)\right\rangle \cong \Sigma_{3}$.

Turning to the extension $E_{3}$, we can extend $\tau, \tau^{\prime}$ to $\Gamma^{1}(P)$ by letting them act identically on $x_{14}$. Then a simple calculation shows $\left\langle(\sigma, \tau),\left(\sigma^{\prime}, \tau^{\prime}\right)\right\rangle \leqslant C_{\chi}$. We note that $\left[E_{3}\right]=\left[E_{2}\right]$ considered as an element of $H_{\bar{\chi}}^{2}\left(V ; \Gamma^{1}(P)\right)$. This follows from the definition of the cocycle

$$
f: V \times V \rightarrow \Gamma^{1}(P) / \Gamma^{2}(P)
$$

for $\left[E_{2}\right]$. Recall that given a set theoretic section $s: V \rightarrow P / \Gamma^{2}(P)$ then

$$
s(a) s(b)=i_{2}(f(a, b)) s(a b)
$$

If we use one of the sections not involving the center, then it lifts to a section $\tilde{s}$ : $V \rightarrow P$ and the corresponding cocycle $\tilde{f}: V \times V \rightarrow \Gamma^{1}(P)$ is a lift of $f$. (For example using the ordered basis $\left(\bar{x}_{12}, \bar{x}_{23}, \bar{x}_{34}\right)$ for $V$ let $\tilde{s}\left(\bar{x}_{12}\right)=x_{12}, \tilde{s}\left(\bar{x}_{23}\right)=x_{23}, \tilde{s}\left(\bar{x}_{34}\right)=$ $x_{34}$. For products $w \in V$ let $\tilde{s}(w)=w^{\prime}$ where $w^{\prime}$ is ordered lexicographically. Thus if $w=\bar{x}_{34} \bar{x}_{23} \bar{x}_{12}$ then $w^{\prime}=x_{12} x_{23} x_{34}$. Hence

$$
\tilde{s}\left(\bar{x}_{34} \bar{x}_{23}\right) \tilde{s}\left(\bar{x}_{12}\right)=i_{3}\left(\tilde{f}\left(\bar{x}_{34} \bar{x}_{23}, \bar{x}_{12}\right)\right) \tilde{s}\left(\bar{x}_{34} \bar{x}_{23} \bar{x}_{12}\right)
$$

implies $\tilde{f}\left(\bar{x}_{34} \bar{x}_{23}, \bar{x}_{12}\right)=x_{13} x_{24}$ In general we observe that no two-fold products among the elements of $\tilde{s}(V)$ involve the center i.e., two-fold products among the elements $x_{12}, x_{23}, x_{34}$ are on the first and second diagonals, not in the center.)

This means $(\sigma, \tau),\left(\sigma^{\prime}, \tau^{\prime}\right)$ fix the extension class $\left[E_{3}\right]$ and hence define automorphisms of $P$ not just $P / \Gamma^{1}(P)$.

4. Let $p$ be an odd prime and let

$P=\langle a, b, c, d| a^{p}=b^{p}=c^{p}=d^{p}=1, c=(b, a), d=(c, a)$, other commutators trivial $\rangle$

We shall study $\operatorname{Aut}(P)$ for $p=5$, the smallest prime for which $P$ is regular. We consider the extensions of $V=P / \Gamma^{1}(P)=\langle\bar{a}, \bar{b}\rangle$

$$
\begin{gathered}
{\left[E_{2}\right]: \quad N_{2}:=\Gamma^{1}(P) / \Gamma^{2}(P) \rightarrow P / \Gamma^{2}(P) \rightarrow V} \\
{\left[E_{3}\right]: \quad N_{3}:=\Gamma^{1}(P) \rightarrow P \rightarrow V}
\end{gathered}
$$

Since $\Gamma^{3}(P)=1$, we also have

$$
\left[\widetilde{E}_{2}\right]: \quad \widetilde{N}_{3}:=\Gamma^{2}(P) \rightarrow \Gamma^{1}(P) \rightarrow N_{2}
$$

Each $N$ is Abelian:

$$
N_{2}=\langle\bar{c}\rangle, \quad N_{3}=\langle c, d\rangle, \quad \widetilde{N}_{3}=\langle d\rangle
$$


Noting that $E_{3}$ has non-trivial twisting we apply the algorithm of Section 4 to study $\operatorname{Aut}(P)$.

Claim: The semi-simple quotient of $\mathbf{F}_{p}(A u t(P))$ is $\mathbf{F}_{p}(\mathbf{Z} / 4)^{2}$.

Proof. (sketch)

Extension $E_{2}$ has trivial twisting and is quite similar to that of Example 1. Using the same notation and arguing analogously we can determine the extension class $\left[E_{2}\right]=x y \otimes z \in H^{2}\left(V, N_{2}\right)$. Hence

$$
C_{\left[E_{2}\right]}=\left\langle(A, B) \in G L_{2}\left(\mathbf{F}_{p}\right) \times G L_{1}\left(\mathbf{F}_{p}\right) \mid B=\operatorname{det}(A)\right\rangle
$$

Extension $\widetilde{E}_{2}$ splits, $\Gamma^{1}(P)=\widetilde{N}_{2} \times N_{2}=\langle c, d\rangle$. Thus every $\tau \in \operatorname{Aut}\left(N_{2}\right)$ lifts to $\operatorname{Aut}\left(\Gamma^{1}(P)\right)$ and we proceed to study extension $E_{3}$.

First we determine

$$
C_{\chi}=\left\langle(\sigma, \tau) \in \operatorname{Aut}(V) \times \operatorname{Aut}\left(N_{3}\right) \mid \chi \sigma=c_{\bar{\tau}} \chi\right\rangle
$$

The action of $V$ on $N_{2}$ is given by $\chi(\bar{x}): c \mapsto c+d, d \mapsto d, \chi(\bar{y})=i d$. Hence $\operatorname{ker}(\chi)=\langle\bar{y}\rangle$. Since $\sigma: \operatorname{ker}(\chi) \rightarrow \operatorname{ker}(\chi)$, it must have the form

$$
\sigma=\left(\begin{array}{cc}
k & 0 \\
m & n
\end{array}\right) \in G L_{2}\left(\mathbf{F}_{p}\right)
$$

Now solving $\chi \sigma=c_{\bar{\tau}} \chi$ where

$$
\tau=\left(\begin{array}{cc}
s & t \\
u & v
\end{array}\right) \in G L_{2}\left(\mathbf{F}_{p}\right)
$$

we find

$$
\left(\begin{array}{ll}
1 & 0 \\
k & 1
\end{array}\right)=\left(\begin{array}{cc}
1+t v / \Delta & t^{2} / \Delta \\
v^{2} / \Delta & 1-t v / \Delta
\end{array}\right)
$$

where $\Delta=\operatorname{det}(\tau)$. Thus $t=0$ and $k=v / s$. Hence

$$
C_{\chi}=\left\{\left(\begin{array}{cc}
k & 0 \\
m & n
\end{array}\right) \times\left(\begin{array}{cc}
s & 0 \\
u & v
\end{array}\right) \mid k=v / s\right\}
$$

To determine $\left.\operatorname{Im}(\rho)=C_{(\chi)}\right)_{\left[E_{3}\right]}$ it is easier in this case to observe that $|\operatorname{Aut}(P)|=$ $4^{2} 5^{5}$ (using Magma) and argue directly instead of using the extension class. First $\operatorname{ker}(\rho)$ contains a subgroup of order $5^{3}$ generated by the inner automorphisms of order $5,\left\{c_{b}, c_{c}\right\} \leqslant \operatorname{ker}(\rho)$ together with the automorphism $\phi: \phi(b)=b d^{-1}, \phi=i d$ on $a, c, d$. Next we show that $\operatorname{Im}(\rho)$ contains a subgroup of order $4^{2} 5^{2}$ which must equal $\operatorname{Im}(\rho)$.

To complete the proof of the claim we note that the subgroup of order $5^{2}$ is normal. 


\section{References}

[1] A. Adem, D. Karagueuzian, J. Minac: On the cohomology of Galois groups determined by Witt rings, Adv. Math., 148 (1999), 105-160.

[2] D. Benson, M. Feshbach: Stable splittings of classifying spaces of finite groups, Topology, 31 (1992), 157-176.

[3] W. Browder: Surgery on simply-connected manifolds, Ergebnisse der Mathematik, 65, Springer-Verlag, Berlin Heidelberg New York, 1972.

[4] J. Buckley: Automorphism groups of isoclinic p-groups, J. London Math. Soc, 12 (1975), 37-44.

[5] J. Dieudonné: La Geometrie des Groupes Classiques, Ergebnisse der Mathematik, 5, Springer-Verlag, Berlin Heidelberg New York, 1963.

[6] L. Dickson: Linear Groups, Dover Publications, New York, 1958.

[7] J. Dietz: Automorphisms of p-groups given as cyclic-by-elementary Abelian central extensions, J. Algebra, 242 (2001), 417-432.

[8] D. Gorenstein: Finite Groups, Chelsea Publications, New York, 1980.

[9] J. Martino, S. Priddy: The complete stable splitting for the classifying space of a finite group, Topology, 31 (1992), 143-156.

[10] J. Martino, S. Priddy: Stable homotopy classification of $B G_{p}^{\wedge}$, Topology, 34, (1995), 633-649.

[11] J. Martino, S. Priddy, J. Douma: On stably decomposing products of classifying spaces, Math. Zeit., 235 (2000), 435-453.

[12] S. MacLane: Homology, Die Grundlehren der Mathemaischen Wissenschafen in Einzeldarstellungen, 114, Springer Verlag, Berlin Heidelberg New York, 1963.

[13] J.-P. Serre: Linear Representations of Finite Groups, Graduate Texts in Mathematics, 42, Springer-Verlag, Berlin Heidelberg New York, 1977.

[14] C. Wells: Automorphisms of group extensions, Trans. Amer. Math. Soc., 155 (1971), 189-194.

[15] D. Winter: The automorphism group of an extraspecial p-group, Rocky Mount. J. Math., 2 (1972), 159-168.

This article may be accessed via WWW at http://www.rmi.acnet.ge/hha/ or by anonymous ftp at

ftp://ftp.rmi.acnet.ge/pub/hha/volumes/2003/n1a3/v5n1a3.(dvi,ps,pdf) 
John Martino martino@wmich.edu

Department of Mathematics

Western Michigan University

Kalamazoo, MI 49008

U.S.A.

Stewart Priddy priddy@math.northwestern.edu

Department of Matheamtics

Northwestern University

Evanston, IL 60208

U.S.A. 